\title{
Modelling of a Human Driver's Interaction with Vehicle Automated Steering using Cooperative Game Theory
}

\author{
Xiaoxiang $\mathrm{Na}$ and David Cole
}

\begin{abstract}
The introduction of automated driving systems raised questions about how the human driver interacts with the automated system. Non-cooperative game theory is increasingly used for modelling and understanding such interaction, while its counterpart, cooperative game theory is rarely discussed for similar applications despite it may be potentially more suitable. This paper describes the modelling of a human driver's steering interaction with an automated steering system using cooperative game theory. The distributed Model Predictive Control approach is adopted to derive the driver's and the automated steering system's strategies in a Pareto equilibrium sense, namely their cooperative Pareto steering strategies. Two separate numerical studies are carried out to study the influence of strategy parameters, and the influence of strategy types on the driver's and the automated system's steering performance. It is found that when a driver interacts with an automated steering system using a cooperative Pareto steering strategy, the driver can improve his/her performance in following a target path through increasing his/her effort in pursuing his/her own interest under the driver-automation cooperative control goal. It is also found that a driver's adoption of cooperative Pareto steering strategy leads to a reinforcement in the driver's steering angle control, compared to the driver's adoption of non-cooperative Nash strategy. This in turn enables the vehicle to return from a lane-change maneuver to straight-line driving swifter.
\end{abstract}

Index Terms-Driver, automated driving system, modelling, cooperative game theory.

\section{INTRODUCTION}

$\mathrm{T}$ HE past decade has seen many advances in automated driving technology. Sophisticated systems capable of performing various aspects of driving tasks independently of human drivers have been developed [1]. Such automated driving systems are believed to possess great potential to reduce traffic accidents and improve social productivity [2]. However, from the point of views of liability and safety, human drivers are expected to retain their control of vehicle during driving [3]. Consequently, there can be situations where the human driver and the automated driving system apply control actions to a vehicle simultaneously. Consider that a human driver is driving

Manuscript received $\mathrm{xx}$ Xxx, $\mathrm{xxxx}$ revised $\mathrm{xx}$ Xxx $\mathrm{xxxx}$; accepted $\mathrm{xx}$ Xxx $\mathrm{xxxx}$. Date of publication $\mathrm{xx} \mathrm{Xxx}, \mathrm{xxxx}$; data of current version $\mathrm{xx} \mathrm{Xxx}, \mathrm{xxxx}$. This paper was recommended by Associate Editor X. Xxx. (Corresponding author: Xiaoxiang $\mathrm{Na}$.)

The authors are with the Department of Engineering, University of Cambridge, CB2 1PZ, Cambridge, U.K. (e-mail: xnhn2@cam.ac.uk; djc13@cam.ac.uk).

Digital Object Identifier xx.xx a vehicle equipped with an automated steering system that can apply steering actions independently of the driver, such as the Active Front Steering (AFS) system described in [4] which features superimposing a steering angle upon the driver's steering wheel angle through a planetary gear apparatus. Now assume that a pedestrian suddenly runs into the road in front of the vehicle. On detecting the pedestrian, the automated steering system may decide to steer around the pedestrian based on some prescribed hazard evaluation logic, e.g. the one proposed in [5]. However, the driver may decide to decelerate the vehicle and meanwhile keep the vehicle travelling straight ahead since the driver may think the pedestrian will run across the road very quickly. As a result, the objective of the driver and that of the automated steering system diverge. This may cause the driver and the automated steering system to compete via steering. For example, the automated steering system steers the vehicle leftwards to avoid the pedestrian while the driver steers in the opposite direction to keep the vehicle travelling straight ahead. A possible consequence is that the vehicle eventually follows a path neither desirable to the automated steering system nor to the human driver. In the worst case, this may lead to a collision with the pedestrian. The situation described above suggests the significance of understanding the interaction between human drivers and vehicle automated driving systems and predicting the impacts of the interaction on vehicle directional responses.

Research on the interaction between human drivers and automated driving systems currently relies heavily on experimental approaches which are time-consuming and expensive. A further issue may arise from automotive manufacturers' continuing efforts into the development of new automated driving technology. A new development may expand the dimensions of present problems so that existing experimental-based solutions and knowledge become less effectual. An alternative approach is to establish a mechanistic model of human driver's interaction with automated driving systems. Such a mechanistic model offers the capability of predicting and interpreting human driver's reaction to automation intervention so that systematic optimization of present or future automated driving technology, e.g., sharing control [6], platooning [7], and parallel driving [8], becomes a possibility.

Dynamic game theory provides a mechanistic basis for understanding the driver-automation interaction. From a dynamic game perspective, the human driver and the automated driving system are game players who make decisions 
independently and act with each other in a repeated manner. As a result, each one's action will influence the other's well-being. In the situation described above, the human driver's and the automated driving system's well-beings can be defined as their individual path-following interest. A dynamic game can be either non-cooperative or cooperative, depending on how game players take into account each other's interest [9]. In a non-cooperative game, each player focuses on pursuing their own interest without caring about other players' interest. In contrast, players in a cooperative game tend to have a sense of collective responsibility and attempt to reach a binding agreement of interests [10].

In recent years, there are increasing published reports of non-cooperative game theory applied in driver-vehicle dynamics. Ma and Peng [11] developed a non-cooperative game framework to evaluate the worst-case performance of a car under simultaneous control a driver and a vehicle stability controller. Tamaddoni et al. [12] used a non-cooperative game framework to develop a vehicle stability controller that accounts for driver's steering input to reduce vehicle lateral and yaw motions in lane change maneuvers. Dextreit and Kolmanovsky [13] reported an algorithm for energy management of hybrid vehicles using non-cooperative game theory to compensate drivers' inattention to eco-driving. Flad et al. [14] developed a steering assistance system where the driver's steering wheel torque was predicted based on the solution to a non-cooperative game between the driver and the steering controller. $\mathrm{Na}$ and Cole [15] proposed a non-cooperative game-theoretic scheme for representing a driver's steering behavior in response to an active steering controller in a collision avoidance scenario. Most recently, Ji et al. developed a non-cooperative game framework for implementing shared control between a driver and an angle angle-overlay steering system [16], and with a torque-overlay steering system [17], where the influence of different driver steering strategies were considered.

In comparison to the ever-growing non-cooperative-gameoriented applications in driver-vehicle dynamics, little treatment has yet been given to its cooperative game counterpart. One instance of the limited work is by the authors [18] who outlined a scheme potentially viable in representing a human driver's interaction with vehicle automated steering using cooperative game theory. The present paper expands the research described in [18] by incorporating a detailed formulation of the mathematical derivation of the driver's and the automated steering system's control strategies in a Pareto equilibrium sense, followed by two separate numerical studies on the influence of parameters and strategy types on the driver's and the automated system's control behavior.

The remainder of the paper is organized as follows. Section II recaps the scheme used for discussing the cooperative-game interaction between a human driver and a vehicle automated driving system. Section III formulates the analytical derivation of the driver's and the automated steering system's cooperative Pareto steering strategies. A numerical study is then carried out to examine the influence of key parameters in the strategies on

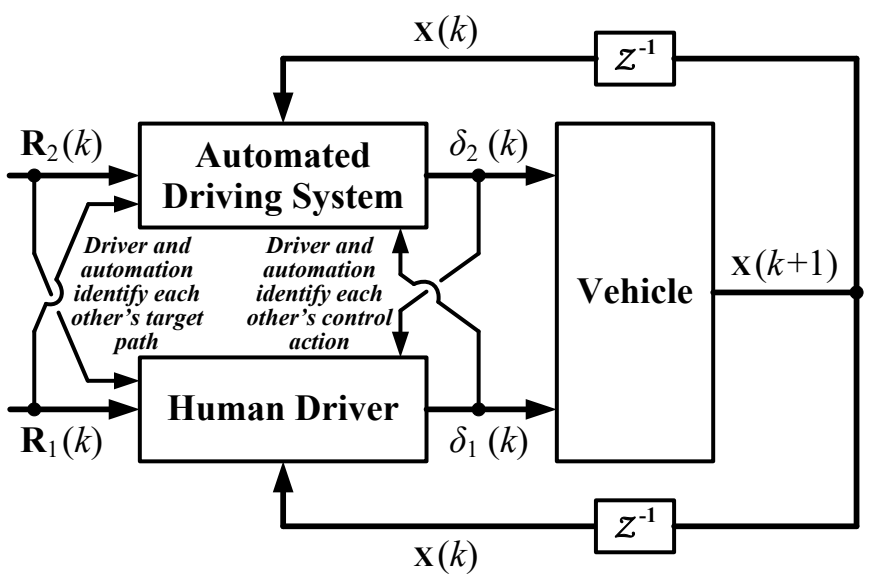

Fig. 1. Scheme for cooperative-game interaction between human driver and automated driving system.

the controllers' steering behavior and vehicle response. Section IV describes a case study that examines the difference between a driver's adoption of a cooperative Pareto steering strategy and his/her adoption of a non-cooperative Nash strategy. Section VI draws conclusions and suggests future work.

\section{SCHEME FOR COOPERATIVE-GAME INTERACTION BETWEEN HuMAN DRIVER AND AUTOMATED DRIVING SYSTEM}

Fig. 1 shows the scheme for cooperative-game interaction between a human driver and a vehicle automated driving system. The scheme describes how a "human driver" may interact with an "automated driving system" in a circumstance that each acts by coordinating its own interest with the other's interest. The "automated driving system" exhibited in Fig. 1 can be an automated steering system for collision avoidance, e.g. [5], or an active accelerator pedal for eco-driving, e.g. [19], etc.

Under this scheme, the automated driving system determines its control action $\delta_{2}(k)$ at time step $k$ by taking into account its own interest $\mathbf{R}_{2}(k)$ as well as the driver's control action $\delta_{1}(k)$ and the driver's interest $\mathbf{R}_{1}(k)$. When an automated steering system is concerned, $\delta_{2}(k)$ is the steering angle applied by the system at time step $k$, and $\delta_{1}(k)$ is the steering angle generated by the driver. $\mathbf{R}_{2}(k)$ and $\mathbf{R}_{1}(k)$ are target road paths planned by the automated steering system and the driver at time step $k$, respectively. Hence, the automated system's control strategy can be conceptually expressed as:

$$
\delta_{2}(k)=f_{2}\left(\mathbf{x}(k), \mathbf{R}_{2}(k), \delta_{1}(k), \mathbf{R}_{1}(k)\right)
$$

Here $f_{2}(\bullet)$ denotes the rule that maps $\mathbf{x}(k), \mathbf{R}_{2}(k), \delta_{1}(k)$ and $\mathbf{R}_{1}(k)$ to the automated system's control action $\delta_{2}(k)$. $\mathbf{x}(k)$ represents vehicle state vector consisting of motion states such as lateral velocity $v(k)$ and yaw rate $\omega(k)$. More details on $\mathbf{x}(k)$ will be provided in the next section. $\mathbf{x}(k)$ can be measured or estimated in most modern vehicles [20]. Driver's control action $\delta_{1}(k)$ can be measured using low-cost sensors. Driver's target $\mathbf{R}_{1}(k)$ can be identified by using appropriate 
driver intention detection algorithms [21].

In practice it is possible that a human driver is able to identify some characteristics of the automated system. Specifically, the automated steering system's target path $\mathbf{R}_{2}(k)$ may be imparted to the driver via some onboard display, as discussed in [3]. Its control action $\delta_{2}(k)$ may be communicated to the driver either through a display in a similar way, or through haptic feedback. Consequently, the driver may be able to derive his/her own control strategy by accounting for $\mathbf{R}_{2}(k)$ and $\delta_{2}(k)$, as depicted in Fig. 1. Hence, the human driver's control strategy can be conceptually expressed as:

$$
\delta_{1}(k)=f_{1}\left(\mathbf{x}(k), \mathbf{R}_{1}(k), \delta_{2}(k), \mathbf{R}_{2}(k)\right)
$$

where $f_{1}()$ denotes the mapping rule.

\section{Derivation of Control Strategies In CoOperative- GAME INTERACTION}

In this section, analytical derivation of the driver's and the automated driving system's control strategies (1) and (2) will be discussed in the context of a human driver interacting with an automated steering system. A numerical analysis will then be carried out to investigate the influence of significant control strategy parameters on the driver's and the automated system's steering actions and the resultant vehicle motions.

\section{A. Driver-Vehicle Dynamics Model}

The automated steering system concerned in this paper involves a mechanism that allows steering angles to be applied to the vehicle independent of driver steering wheel action. Such a mechanism can be implemented through the Active Front Steering (AFS) system described in [4]. In such a system, the steering angle of vehicle front wheels is the summation of the angle applied by the driver $\delta_{1}(k)$ and the angle exerted by the AFS actuator $\delta_{2}(k)$. Hence, the dynamic evolution of vehicle state $\mathbf{x}(k)$ can be described using the following state-space expression:

$$
\begin{aligned}
\mathbf{x}(k+1) & =\mathbf{A x}(k)+\mathbf{B}_{1} \delta_{1}(k)+\mathbf{B}_{2} \delta_{2}(k) \\
\mathbf{z}(k) & =\mathbf{C x}(k)
\end{aligned}
$$

The vehicle state vector $\mathbf{x}(k)$ at time step $k$ comprises vehicle lateral velocity $v(k)$, yaw rate $\omega(k)$, lateral displacement $y(k)$, lateral displacement integral $y_{\text {int }}(k)$, and yaw angle $\psi(k)$, i.e. $\mathbf{x}(k)=\left\{\begin{array}{llllll}v(k) & \omega(k) & y(k) & y_{\text {int }}(k) & \psi(k)\end{array}\right\}^{\mathrm{T}}$. It can be seen from (3) that the evolution of $\mathbf{x}(k)$ is driven by the driver's steering angle $\delta_{1}(k)$ and the vehicle's steering angle $\delta_{2}(k)$. $\mathbf{A}$ is the state matrix, $\mathbf{B}_{1}$ and $\mathbf{B}_{2}$ are input vectors associated with $\delta_{1}(k)$ and $\delta_{2}(k)$, respectively. For the sake of simplicity, the dynamics of the vehicle in this paper are represented using a linear time-invariant single-track model [18]. Therefore, $\mathbf{A}, \mathbf{B}_{1}$ and $\mathbf{B}_{2}$ are time-invariant. $\mathbf{z}(k)$ is the output vector which is set to $\mathbf{z}(k)=\left\{\begin{array}{lll}y(k) & y_{\text {int }}(k) & \psi(k)\end{array}\right\}^{\mathrm{T}}$. It represents the vehicle's position and orientation. $\mathbf{C}$ is the output matrix that converts $\mathbf{x}(k)$ to $\mathbf{z}(k)$.

\section{B. Pareto Strategies}

In a theoretic game, an equilibrium represents a situation where no player is willing to change their strategy unilaterally since they will not gain any more by doing so [9]. In the context of a cooperative game, such an equilibrium is called a "Pareto equilibrium". Its counterpart in a non-cooperative game is the famous "Nash equilibrium" [22]. The strategy of a player at a Pareto equilibrium is therefore called his/her cooperative Pareto strategy.

On modelling of human drivers' steering behavior, the Model Predictive Control (MPC) method is a natural choice since it is consistent with the "internal model" hypothesis widely adopted in neuroscience [23]. The suitability of using MPC for modelling human drivers' steering behavior was demonstrated in several separated research, for example [24] and [25]. In both studies, the authors developed MPC-based driver steering control models and compared the optimal steering strategies derived from the driver models to measured human driver steering angles. It was found in both studies that the optimal steering strategies could represent the steering behavior of a variety of human drivers with different driving skills and experience. When the problem involves a human driver and an automated steering system who make decisions independently and apply steering actions simultaneously, distributed MPC, an extension of MPC to multi-agent applications becomes a possible option. The theoretical link between distributed MPC and dynamic games was established by Rawlings and Mayne [26], where the construction of game players' optimization problems in both non-cooperative and cooperative cases were described.

In this paper, the distributed MPC approach is adopted for deriving the analytical expressions of the driver's and the automated system's steering strategies in a Pareto equilibrium sense. This generally involves three steps: 1) construction of the human driver's and the automated steering system's cost functions, 2) establishment of their prediction equations, and 3) derivation of their cooperative Pareto steering strategies by optimizing their cost functions subject to their prediction equations.

\section{1) Construction of Cost Functions}

Regarding the interaction between a human driver and an automated steering system, the driver and the automated system have individual target road paths $\mathbf{R}_{1}(k)$ and $\mathbf{R}_{2}(k)$, as described in Fig. 1. $\mathbf{R}_{1}(k)$ can be expressed as a sequence of vectors from the driver's target vehicle orientation at $k+1$ step, i.e. $\mathbf{r}_{1}(k+1)$ up to the driver's target vehicle orientation at $k+$ $N_{1}$ step, i.e. $\mathbf{r}_{1}\left(k+N_{1}\right)$, where $N_{1}$ is called the driver's preview horizon:

$$
\mathbf{R}_{1}(k)=\left\{\begin{array}{c}
\mathbf{r}_{1}(k+1) \\
\mathbf{r}_{1}(k+2) \\
\mathbf{r}_{1}\left(k+N_{1}\right)
\end{array}\right\}
$$

The value of $N_{1}$ is closely associated with the distance that a 
human driver can look ahead. Each $\mathbf{r}_{1}(k+j)$ where $j=1,2, \ldots$, $N_{1}$ consists of three elements: target lateral displacement $r_{1}^{y}(k+j)$, target lateral displacement integral $r_{1}^{y_{\text {int }}}(k+j)$, and target yaw angle $r_{1}^{\psi}(k+j)$, that is:

$$
\mathbf{r}_{1}(k+j)=\left\{\begin{array}{c}
r_{1}^{y}(k+j) \\
r_{1}^{y_{\text {int }}}(k+j) \\
r_{1}^{\mu}(k+j)
\end{array}\right\}, \text { for } j=1,2, \ldots, N_{1}
$$

The driver's key interest at each time step $k$ can be described as to minimize his/her road path tracking error $\mathbf{E}_{1}(k)$, where $\mathbf{E}_{1}(k)=\mathbf{Z}_{1}(k)-\mathbf{R}_{1}(k)$. The term $\mathbf{Z}_{1}(k)$ represents the future path that the driver predicts at time step $k$ up to his/her preview horizon $N_{1} . \mathbf{Z}_{1}(k)$ holds the following format:

$$
\mathbf{Z}_{1}(k)=\left\{\begin{array}{c}
\mathbf{z}(k+1) \\
\mathbf{z}(k+2) \\
\mathbf{z}\left(k+N_{1}\right)
\end{array}\right\}
$$

Derivation of $\mathbf{Z}_{1}(k)$ follows an iterative approach towards the driver-vehicle dynamics equation (3) for $N_{1}$ steps ahead. This approach will be described in detail later. Readers are suggested to bear in mind at the moment that $\mathbf{Z}_{1}(k)$ is independent of the driver's target road path $\mathbf{R}_{1}(k)$ but is influenced by the driver's steering actions in the future up to the driver's preview horizon $N_{1} . \mathbf{E}_{1}(k)$ contains essentially the difference between the driver's target vehicle orientations and his/her predicted vehicle orientations at each future step, up to the driver's preview horizon $N_{1}$.

Similarly, the automated steering system's target path $\mathbf{R}_{2}(k)$ comprises its own target vehicle orientations up to its preview horizon $N_{2}$. The automated system's key interest at time step $k$ is to minimize its path tracking error $\mathbf{E}_{2}(k)=\mathbf{Z}_{2}(k)-\mathbf{R}_{2}(k)$, where $\mathbf{Z}_{2}(k)$ is the path predicted by the automated steering system up to its preview horizon $N_{2}$.

Since in a cooperative game, each game player tends to take care of all the other players' interests, the cost function that the driver minimizes during his/her cooperative interaction with the automated steering system can be constructed as:

$$
\begin{aligned}
J_{1}(k) & =\rho_{1} \mathbf{E}_{1}(k)^{\mathrm{T}} \mathbf{Q}_{1} \mathbf{E}_{1}(k)+\rho_{2} \mathbf{E}_{2}(k)^{\mathrm{T}} \mathbf{Q}_{2} \mathbf{E}_{2}(k) \\
& +\rho_{1} \mathbf{U}_{1}(k)^{\mathrm{T}} \mathbf{U}_{1}(k)
\end{aligned}
$$

In (4) $\mathbf{U}_{1}(k)$ is a vector of driver's control actions from current steering angle $\delta_{1}(k)$ up to the future angle $\delta_{1}\left(k+N_{1}-1\right)$, that is

$$
\mathbf{U}_{1}(k)=\left\{\begin{array}{c}
\delta_{1}(k) \\
\delta_{1}(k+1) \\
\delta_{1}\left(k+N_{1}-1\right)
\end{array}\right\} .
$$

(4) implies that the driver aims at minimizing both the driver's own tracking error $\mathbf{E}_{1}(k)$ and the automated steering system's tracking error $\mathbf{E}_{2}(k)$. $\mathbf{Q}_{1}$ is a diagonal matrix that weights $\mathbf{E}_{1}(k) \cdot \mathbf{Q}_{1}$ can be expressed as:

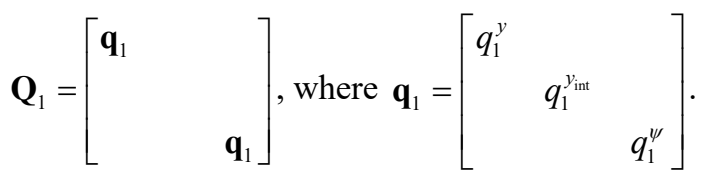

$q_{1}^{y}, q_{1}^{y_{\text {int }}}$ and $q_{1}^{\psi /}$ are time-invariant weights that weigh respectively the lateral displacement error, lateral displacement error integral and yaw angle error involved in $\mathbf{E}_{1}(k) \cdot \mathbf{Q}_{2}$ weights $\mathbf{E}_{2}(k)$ in an identical way. $\mathbf{Q}$, can be expressed as:

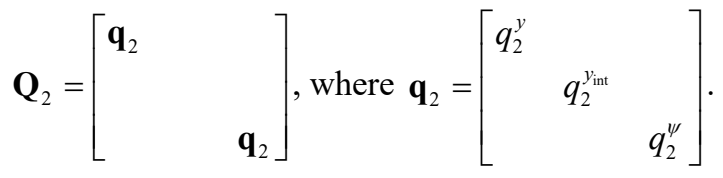

$\mathbf{Q}_{1}$ and $\mathbf{Q}_{2}$ may differ from each other as the driver and the automated steering system may have different opinions in how strongly the tracking errors shall be weighted. $\rho_{1}$ and $\rho_{2}$ are parameters used to specify the relative weights between $\mathbf{E}_{1}(k)$ and $\mathbf{E}_{2}(k)$. The driver's cost function (4) can be then simplified as:

$$
J_{1}(k)=\mathbf{E}(k)^{\mathrm{T}} \mathbf{Q E}(k)+\rho_{1} \mathbf{U}_{1}(k)^{\mathrm{T}} \mathbf{U}_{1}(k)
$$

where

$$
\mathbf{E}(k)=\left\{\begin{array}{l}
\mathbf{E}_{1}(k) \\
\mathbf{E}_{2}(k)
\end{array}\right\}=\left\{\begin{array}{l}
\mathbf{Z}_{1}(k)-\mathbf{R}_{1}(k) \\
\mathbf{Z}_{2}(k)-\mathbf{R}_{2}(k)
\end{array}\right\} \text { and } \mathbf{Q}=\left[\begin{array}{cc}
\rho_{1} \mathbf{Q}_{1} & \mathbf{0} \\
\mathbf{0} & \rho_{2} \mathbf{Q}_{2}
\end{array}\right] .
$$

Similarly, the cost function that the automated steering system attempts to minimize can be constructed as:

$$
J_{2}(k)=\mathbf{E}(k)^{\mathrm{T}} \mathbf{Q E}(k)+\rho_{2} \mathbf{U}_{2}(k)^{\mathrm{T}} \mathbf{U}_{2}(k)
$$

It can be seen from (5) and (6) that both the driver and the automated steering system aim at minimizing $\mathbf{E}_{1}(k)$ and $\mathbf{E}_{2}(k)$ collectively. However, their steering angles may differ due to the existence of relative weights $\rho_{1}$ and $\rho_{2}$.

\section{2) Establishment of Prediction Equations}

The predicted path $\mathbf{Z}_{1}(k)$ held by the driver is derived by iterating the driver-vehicle dynamics equation (3). This is started by deriving the output vector at time step $k+2$, i.e. $\mathbf{z}(k+2)$ following the procedure described below:

$$
\begin{aligned}
\mathbf{z}(k+2) & =\mathbf{C x}(k+2) \\
& =\mathbf{C A x}(k+1)+\mathbf{C} \mathbf{B}_{1} \delta_{1}(k+1)+\mathbf{C} \mathbf{B}_{2} \delta_{2}(k+1) \\
& =\mathbf{C A}^{2} \mathbf{x}(k)+\mathbf{C A B} \delta_{1}(k)+\mathbf{C A B} \delta_{2}(k) \\
& +\mathbf{C B}_{1} \delta_{1}(k+1)+\mathbf{C B}_{2} \delta_{2}(k+1)
\end{aligned}
$$

By continuing the iteration, the future output vectors from $\mathbf{z}(k+3)$ up to $\mathbf{z}\left(k+N_{1}\right)$ can be derived. Hence, the driver's predicted path $\mathbf{Z}_{1}(k)$ can be expressed as:

$$
\mathbf{Z}_{1}(k)=\boldsymbol{\Psi}_{1} \mathbf{x}(k)+\boldsymbol{\Theta}_{1} \mathbf{U}_{1}(k)+\boldsymbol{\Omega}_{1} \mathbf{U}_{2}(k)
$$

where 


$$
\begin{aligned}
& \mathbf{Z}_{1}(k)=\left\{\begin{array}{c}
\mathbf{z}(k+1) \\
\mathbf{z}(k+2) \\
\mathbf{z}\left(k+N_{1}\right)
\end{array}\right\}, \mathbf{U}_{1}(k)=\left\{\begin{array}{c}
\delta_{1}(k) \\
\delta_{1}(k+1) \\
\\
\delta_{1}\left(k+N_{1}-1\right)
\end{array}\right\}, \\
& \mathbf{U}_{2}(k)=\left\{\begin{array}{c}
\delta_{2}(k) \\
\delta_{2}(k+1) \\
\delta_{2}\left(k+N_{1}-1\right)
\end{array}\right\}, \boldsymbol{\Psi}_{1}=\left[\begin{array}{c}
\mathbf{C A} \\
\mathbf{C A}{ }^{2} \\
\mathbf{C A}^{N_{1}}
\end{array}\right], \\
& \boldsymbol{\Theta}_{1}=\left[\begin{array}{ccc}
\mathbf{C B}_{1} & \mathbf{0} & \mathbf{0} \\
\mathbf{C A B}_{1} & \mathbf{C B}_{1} & \mathbf{0} \\
& & \\
\mathbf{C A}^{N_{1}-1} \mathbf{B}_{1} & \mathbf{C A}^{N_{1}-2} \mathbf{B}_{1} & \mathbf{C B}_{1}
\end{array}\right] \text {, and } \\
& \boldsymbol{\Omega}_{1}=\left[\begin{array}{ccc}
\mathbf{C B}_{2} & \mathbf{0} & \mathbf{0} \\
\mathbf{C A B}_{2} & \mathbf{C B}_{2} & \mathbf{0} \\
& & \\
\mathbf{C A}^{N_{1}-1} \mathbf{B}_{2} & \mathbf{C A}^{N_{1}-2} \mathbf{B}_{2} & \mathbf{C B}_{2}
\end{array}\right] .
\end{aligned}
$$

Equation (8) is called the driver's prediction equation.

By following a similar procedure, the automated steering system's prediction equation (9) can be derived as:

$$
\mathbf{Z}_{2}(k)=\boldsymbol{\Psi}_{2} \mathbf{x}(k)+\boldsymbol{\Theta}_{2} \mathbf{U}_{1}(k)+\mathbf{\Omega}_{2} \mathbf{U}_{2}(k)
$$

where

$$
\begin{gathered}
\mathbf{Z}_{2}(k)=\left\{\begin{array}{c}
\mathbf{z}(k+1) \\
\mathbf{z}(k+2) \\
\mathbf{z}\left(k+N_{2}\right)
\end{array}\right\}, \mathbf{U}_{1}(k)=\left\{\begin{array}{c}
\delta_{1}(k) \\
\delta_{1}(k+1) \\
\delta_{1}\left(k+N_{2}-1\right)
\end{array}\right\}, \\
\mathbf{U}_{2}(k)=\left\{\begin{array}{c}
\delta_{2}(k) \\
\delta_{2}(k+1) \\
\delta_{2}\left(k+N_{2}-1\right)
\end{array}\right\}, \boldsymbol{\Psi}_{2}=\left[\begin{array}{c}
\mathbf{C A} \\
\mathbf{C A}^{2} \\
\mathbf{C A}^{N_{2}}
\end{array}\right], \\
\boldsymbol{\Theta}_{2}=\left[\begin{array}{ccc}
\mathbf{C B}_{1} & \mathbf{0} \\
\mathbf{C A B} & \mathbf{C B}_{1} & \mathbf{0} \\
\mathbf{C A}^{N_{2}-1} \mathbf{B}_{1} & \mathbf{C A}^{N_{2}-2} \mathbf{B}_{1} & \mathbf{C B}
\end{array}\right], \text { and } \\
\mathbf{\Omega}_{2}=\left[\begin{array}{rrr}
\mathbf{C B}_{2} & \mathbf{0} & \mathbf{0} \\
\mathbf{C A B}_{2} & \mathbf{C B}_{2} & \mathbf{0} \\
\mathbf{C A}^{N_{2}-1} \mathbf{B}_{2} & \mathbf{C A}^{N_{2}-2} \mathbf{B}_{2} & \mathbf{C B}
\end{array}\right] .
\end{gathered}
$$

It should be noticed that the driver steering angle array $\mathbf{U}_{1}(k)$ and automated steering angle $\mathbf{U}_{2}(k)$ appearing in (8) are both of length $N_{1}$ while in (9) they are both of length $N_{2}$.

\section{3) Derivation of Pareto Steering Strategies}

Continuing to follow Rawlings and Mayne [26], the driver's and the automated steering system's control strategies can be derived by minimizing their respective cost functions (5) and (6), subject to a 'global' prediction equation which combines (8) and (9). However, the global prediction equation cannot be obtained when the driver's steering angle array $\mathbf{U}_{1}(k)$ in (8) has a different dimension from that in (9). Towards this end, the assumption $N_{1}=N_{2}=N$ is made. As a result, the global prediction equation can be obtained as:

$$
\mathbf{Z}(k)=\mathbf{\Psi x}(k)+\boldsymbol{\Theta} \mathbf{U}_{1}(k)+\boldsymbol{\Omega} \mathbf{U}_{2}(k)
$$

where

$$
\begin{aligned}
& \mathbf{Z}(k)=\left\{\begin{array}{l}
\mathbf{Z}_{1}(k) \\
\mathbf{Z}_{2}(k)
\end{array}\right\}, \boldsymbol{\Psi}=\left\{\begin{array}{c}
\boldsymbol{\Psi}_{1} \\
\boldsymbol{\Psi}_{2}
\end{array}\right\}, \boldsymbol{\Theta}=\left\{\begin{array}{c}
\boldsymbol{\Theta}_{1} \\
\boldsymbol{\Theta}_{2}
\end{array}\right\}, \boldsymbol{\Omega}=\left\{\begin{array}{l}
\boldsymbol{\Omega}_{1} \\
\boldsymbol{\Omega}_{2}
\end{array}\right\}, \\
& \mathbf{U}_{1}(k)=\left\{\begin{array}{c}
\delta_{1}(k) \\
\delta_{1}(k+1) \\
\delta_{1}(k+N-1)
\end{array}\right\} \text {, and } \mathbf{U}_{2}(k)=\left\{\begin{array}{c}
\delta_{2}(k) \\
\delta_{2}(k+1) \\
\delta_{2}(k+N-1)
\end{array}\right\} .
\end{aligned}
$$

It should be noted that the assumption $N_{1}=N_{2}=N$ is reasonable since in many applications, the automated steering system was set to have a preview horizon similar to that of a human driver, which is typically between $0.5 \mathrm{~s}$ to $2 \mathrm{~s}$ [24].

Rawlings and Mayne [26] explained that by minimizing the driver's cost function (5) subject to the global prediction equation (10), the driver's optimal steering angle array $\mathbf{U}_{1}^{*}(k)$ can be developed and expressed in the following form:

$$
\mathbf{U}_{1}^{*}(k)=\mathbf{K}_{1}\left\{\begin{array}{c}
\mathbf{x}(k) \\
\mathbf{R}_{1}(k) \\
\mathbf{R}_{2}(k)
\end{array}\right\}+\mathbf{L}_{1} \mathbf{U}_{2}(k)
$$

where $\mathbf{K}_{1}$ and $\mathbf{L}_{1}$ are time-invariant gain matrices which are functions of $\mathbf{A}, \mathbf{B}_{1}, \mathbf{B}_{2}, \mathbf{C}, \mathbf{Q}_{1}, \mathbf{Q}_{2}, \rho_{1}$ and $\rho_{2}$. Due to space limitation, the intermediate algebraic procedures for deriving (11) are omitted. Readers who are interested in the derivation are recommended to go to [27] for details. (12) implies that the driver's optimal steering angle array $\mathbf{U}_{1}^{*}(k)$ that minimizes the driver's cost function (5) depends linearly on the state of the vehicle $\mathbf{x}(k)$, the driver's target path $\mathbf{R}_{1}(k)$, the automated steering system's target path $\mathbf{R}_{2}(k)$, but also on the automated steering system's steering angle array $\mathbf{U}_{2}(k)$. In other words, the driver accounts for the automated steering system's steering angle actions during his/her process of decision-making.

The automated steering system's optimal steering angle array $\mathbf{U}_{2}^{*}(k)$ can be solved in a similar way, that is by optimizing its cost function (6) subject to the global prediction equation (10):

$$
\mathbf{U}_{2}^{*}(k)=\mathbf{K}_{2}\left\{\begin{array}{c}
\mathbf{x}(k) \\
\mathbf{R}_{1}(k) \\
\mathbf{R}_{2}(k)
\end{array}\right\}+\mathbf{L}_{2} \mathbf{U}_{1}(k)
$$

where $\mathbf{K}_{2}$ and $\mathbf{L}_{2}$ are time-invariant gain matrices which are functions of $\mathbf{A}, \mathbf{B}_{1}, \mathbf{B}_{2}, \mathbf{C}, \mathbf{Q}_{1}, \mathbf{Q}_{2}, \rho_{1}$ and $\rho_{2}$. 
At this point, two observations can be made towards the expressions of the driver's optimal steering angle array (11) and the automated steering system's optimal steering angle array (12). First, (11) and (12) agree in format with the driver's and the automated system's conceptual control strategies (1) and (2), respectively. Second, (11) and (12) are coupled in such a way that the driver's optimal steering angle array $\mathbf{U}_{1}^{*}(k)$ depends on the automated steering system's applied steering angle array $\mathbf{U}_{2}(k)$ and so does $\mathbf{U}_{2}^{*}(k)$. The second observation suggests that $\mathbf{U}_{1}^{*}(k)$ and $\mathbf{U}_{2}^{*}(k)$ cannot be solved by merely relying on (11) and (12). Towards this end, the convex iteration approach [26] is adopted, which involves complementing (11) and (12) with two auxiliary equations:

$$
\begin{aligned}
& \mathbf{U}_{1}(k)^{[p+1]}=w_{1} \mathbf{U}_{1}^{*}(k)^{[p]}+\left(1-w_{1}\right) \mathbf{U}_{1}(k)^{[p]} \\
& \mathbf{U}_{2}(k)^{[p+1]}=w_{2} \mathbf{U}_{2}^{*}(k)^{[p]}+\left(1-w_{2}\right) \mathbf{U}_{2}(k)^{[p]}
\end{aligned}
$$

where $p$ denotes the step of convex iteration, and $w_{1}$ and $w_{2}$ are iteration weights that satisfy $w_{1}, w_{2} \in[0,1]$ and $w_{1}+w_{2}=1$. Based on equations from (11) to (14), the optimal steering angle arrays $\mathbf{U}_{1}^{*}(k)^{[p]}$ and $\mathbf{U}_{2}^{*}(k)^{[p]}$ at any step $p$ can be calculated [26]. This paper focuses on a special case where the iteration step $p$ reaches infinity, i.e. $p \rightarrow \infty$. In this case, $\mathbf{U}_{1}^{*}(k)^{[\infty]}$ and $\mathbf{U}_{2}^{*}(k)^{[\infty]}$ will have equilibrium properties [26], that is $\mathbf{U}_{1}^{*}(k)^{[\infty]}$ and $\mathbf{U}_{2}^{*}(k)^{[\infty]}$ will represent respectively the driver's and the automated steering system's steering angle arrays in a Pareto equilibrium sense. Specifically, when $p \rightarrow \infty$, (11) and (12) can be rewritten respectively as:

$$
\begin{aligned}
& \mathbf{U}_{1}^{*}(k)^{[\infty]}=\mathbf{K}_{1}\left\{\begin{array}{c}
\mathbf{x}(k) \\
\mathbf{R}_{1}(k) \\
\mathbf{R}_{2}(k)
\end{array}\right\}+\mathbf{L}_{1} \mathbf{U}_{2}(k)^{[\infty]} \\
& \mathbf{U}_{2}^{*}(k)^{[\infty]}=\mathbf{K}_{2}\left\{\begin{array}{c}
\mathbf{x}(k) \\
\mathbf{R}_{1}(k) \\
\mathbf{R}_{2}(k)
\end{array}\right\}+\mathbf{L}_{2} \mathbf{U}_{1}(k)^{[\infty]}
\end{aligned}
$$

and (13) and (14) becomes:

$$
\begin{aligned}
& \mathbf{U}_{1}(k)^{[\infty]}=w_{1} \mathbf{U}_{1}^{*}(k)^{[\infty]}+\left(1-w_{1}\right) \mathbf{U}_{1}(k)^{[\infty]} \\
& \mathbf{U}_{2}(k)^{[\infty]}=w_{2} \mathbf{U}_{2}^{*}(k)^{[\infty]}+\left(1-w_{2}\right) \mathbf{U}_{2}(k)^{[\infty]}
\end{aligned}
$$

Substituting (18) into (15) to eliminate $\mathbf{U}_{2}(k)^{[\infty]}$ then yields:

$$
\mathbf{U}_{1}^{*}(k)^{[\infty]}=\mathbf{K}_{1}\left\{\begin{array}{c}
\mathbf{x}(k) \\
\mathbf{R}_{1}(k) \\
\mathbf{R}_{2}(k)
\end{array}\right\}+\mathbf{L}_{1} \mathbf{U}_{2}^{*}(k)^{[\infty]}
$$

Substituting (17) into (16) to eliminate $\mathbf{U}_{1}(k)^{[\infty]}$ then yields:

$$
\mathbf{U}_{2}^{*}(k)^{[\infty]}=\mathbf{K}_{2}\left\{\begin{array}{c}
\mathbf{x}(k) \\
\mathbf{R}_{1}(k) \\
\mathbf{R}_{2}(k)
\end{array}\right\}+\mathbf{L}_{2} \mathbf{U}_{1}^{*}(k)^{[\infty]}
$$

Now $\mathbf{U}_{1}^{*}(k)^{[\infty]}$ and $\mathbf{U}_{2}^{*}(k)^{[\infty]}$ can be decoupled by substituting (19) and (20) into one another. This finally gives:

$$
\begin{aligned}
& \mathbf{U}_{1}^{*}(k)^{[\infty]}=\mathbf{K}_{1}^{*}\left\{\begin{array}{c}
\mathbf{x}(k) \\
\mathbf{R}_{1}(k) \\
\mathbf{R}_{2}(k)
\end{array}\right\} \\
& \mathbf{U}_{2}^{*}(k)^{[\infty]}=\mathbf{K}_{2}^{*}\left\{\begin{array}{c}
\mathbf{x}(k) \\
\mathbf{R}_{1}(k) \\
\mathbf{R}_{2}(k)
\end{array}\right\}
\end{aligned}
$$

where

$$
\begin{aligned}
& \mathbf{K}_{1}^{*}=\left[\mathbf{I}-\mathbf{L}_{1} \mathbf{L}_{2}\right]^{-1}\left[\mathbf{K}_{1}+\mathbf{L}_{1} \mathbf{K}_{2}\right], \\
& \mathbf{K}_{2}^{*}=\left[\mathbf{I}-\mathbf{L}_{2} \mathbf{L}_{1}\right]^{-1}\left[\mathbf{K}_{2}+\mathbf{L}_{2} \mathbf{K}_{1}\right],
\end{aligned}
$$

and $\mathbf{I}$ is an identity matrix of appropriate dimension.

It should be noted that the array $\mathbf{U}_{1}^{*}(k)^{[\infty]}$ appearing in (21) comprises $N$ elements from $\delta_{1}^{*}(k)^{[\infty]}$ to $\delta_{1}^{*}(k+N-1)^{[\infty]}$. Any $\delta_{1}^{*}(k+j)^{[\infty]}$ for $j=0,1,2 \ldots, N-1$ denotes the driver's optimal steering angle value at time step $k+j$, which is determined at current time step $k$. In reality, the driver at current time step $k$ can only apply one particular steering angle value to the vehicle. In view of this, the first element in $\mathbf{U}_{1}^{*}(k)^{[\infty]}$, that is $\delta_{1}^{*}(k)^{[\infty]}$ is chosen as the steering angle action that the driver applies. This technique conforms to the 'receding horizon' idea [28] that is well adopted in Model Predictive Control practice. In view of this, the following expression holds for $\delta_{1}^{*}(k)^{[\infty]}$ :

$$
\delta_{1}^{*}(k)^{[\infty]}=\mathbf{K}_{1}^{*}(1,:)\left\{\begin{array}{c}
\mathbf{x}(k) \\
\mathbf{R}_{1}(k) \\
\mathbf{R}_{2}(k)
\end{array}\right\}
$$

where $\mathbf{K}_{1}^{*}(1,:)$ denotes the first row of $\mathbf{K}_{1}^{*}$. Similarly, the automated steering system's optimal steering angle $\delta_{2}^{*}(k)^{[\infty]}$ applied at time step $k$ can be obtained as

$$
\delta_{2}^{*}(k)^{[\infty]}=\mathbf{K}_{2}^{*}(1,:)\left\{\begin{array}{c}
\mathbf{x}(k) \\
\mathbf{R}_{1}(k) \\
\mathbf{R}_{2}(k)
\end{array}\right\}
$$

where $\mathbf{K}_{,}^{*}(1,:)$ denotes the first row of $\mathbf{K}_{\text {, }}^{*}$.

(23) and (24) are respectively the driver's and automated steering system's cooperative Pareto steering strategies. It should be noted that both $\mathbf{K}_{1}^{*}$ and $\mathbf{K}_{2}^{*}$ are time-invariant gain matrices which are functions of vehicle model parameters $\mathbf{A}$, $\mathbf{B}_{1}, \mathbf{B}_{2}$ and $\mathbf{C}$, the two controllers' path-error weighting matrices $\mathbf{Q}_{1}$ and $\mathbf{Q}_{2}$, and cost function relative weights $\rho_{1}$ and $\rho_{2}$. At this point, the question whether a human driver can build up his/her cooperative Pareto steering strategy (23) might raise since it seems less realistic that a human driver can acquire the precise values of $\mathbf{A}, \mathbf{B}_{1}, \mathbf{B}_{2}, \mathbf{C}, \mathbf{Q}_{1}, \mathbf{Q}_{2}, \rho_{1}$ and $\rho_{2}$. Towards this question, the authors think there should not be any reason to exclude the possibility that a human driver may follow the principle of cooperative game theory to develop their steering strategy. However, the driver may not have perfect knowledge of $\mathbf{A}, \mathbf{B}_{1}, \mathbf{B}_{2}, \mathbf{C}, \mathbf{Q}_{1}, \mathbf{Q}_{2}, \rho_{1}$ and $\rho_{2}$. As a result, the steering angle that the driver applies to the vehicle in practice may differ from that calculated using the driver's 
TABLE I

VEHICLE PARAMETERS

\begin{tabular}{lll}
\hline \hline Symbol & Quantity & Value \\
\hline$m$ & vehicle mass & $1840 \mathrm{~kg}$ \\
$I$ & vehicle yaw moment of inertia & $3000 \mathrm{kgm}^{2}$ \\
$l_{a} / l_{b}$ & $\begin{array}{l}\text { distance from vehicle centre of } \\
\text { mass to front axle / rear axle }\end{array}$ & $1.136 / 1.663 \mathrm{~m}$ \\
$C_{f} / C_{r}$ & $\begin{array}{l}\text { cornering stiffness of front axle / } \\
\text { rear axle }\end{array}$ & $116000 / 187000 \mathrm{~N} / \mathrm{rad}$ \\
$G$ & Vehicle overall steering ratio & 15.8 \\
\hline \hline
\end{tabular}

cooperative Pareto strategy (23). Such difference may diminish as the driver increasingly learns the characteristics of the vehicle and the automated steering system via daily driving. It is also feasible to use some noise model to represent the driver's imperfect knowledge of $\mathbf{A}, \mathbf{B}_{1}, \mathbf{B}_{2}, \mathbf{C}, \mathbf{Q}_{1}, \mathbf{Q}_{2}, \rho_{1}$ and $\rho_{2}$, such as the ARMA-based noise model proposed in [29]. Accordingly, the driver's steering angle applied to the vehicle may be determined by deducting the effect of the noise model from the steering angle calculated using the driver's cooperative Pareto strategy (23). Consequently, it would be of significance to investigate and understand the influence of the parameters involved in (23) and (24) on the driver's and the automated steering system's steering angles and vehicle directional responses. This investigation will be carried out through a numerical study in the next subsection.

\section{Numerical Analysis}

In this subsection, the effects of varying four types of parameters are studied: i) cost function relative weights $\rho_{1}$ and $\rho_{2}$, ii) lateral displacement error weights $q_{1}^{y}$ and $q_{2}^{y}$, iii) lateral displacement error integral weights $q_{1}^{y_{\text {int }}}$ and $q_{2}^{y_{\text {int }}}$, and iv) preview horizon $N$. The longitudinal speed of the vehicle is fixed at $20 \mathrm{~m} / \mathrm{s}$ and the simulation time step is fixed at $0.01 \mathrm{~s}$. Values of vehicle parameters used in the simulation are provided in Table I.

The driving scenario employed in the simulation involves a driver and an automated steering system attempting to follow different target paths. Specifically, the driver has a straight-line target path while the automated steering system holds a singlelane-change target path, as described in Table II. It should be noted that such a scenario may be less realistic from a practical point of view since in a cooperative situation the driver and the automated system are very much likely to have similar targets. The main reason for using such a conflicting driving scenario is that it is more helpful for comparing the steering angles given by the driver and the automated steering system.

Fig. 2 (a) illustrates the influence of cost function relative weights $\rho_{1}$ and $\rho_{2}$. In this study, the preview horizon $N$ is set to be 200 steps, i.e. 2.0 s. The driver's and the automated steering system's path-error weights are set to be $q_{1}^{y}=q_{2}^{y}=0.1$, $q_{1}^{\psi \prime}=q_{2}^{\psi}=0$ and $q_{1}^{y_{\text {int }}}=q_{2}^{y_{\text {int }}}=0$. The left plot shows the how the vehicle's center of gravity displaces laterally as it travels ahead. The driver's and the automated steering system's target
TABLE II

DRIVER AND AUTOMATED STEERING SYSTEM TARgET PATHS

\begin{tabular}{c|c|cc|c} 
\\
\hline \hline
\end{tabular}

paths are also provided in this subplot. The right plot shows how the driver's and the automated system's steering angles vary as the vehicle travels ahead. Since the longitudinal speed of the vehicle is fixed at $20 \mathrm{~m} / \mathrm{s}$ throughout the simulation, these two plots are equivalent respectively to the time histories of the vehicle path and the time histories of steering angles. It can be seen that when $\rho_{1}=\rho_{2}=0.5$ (solid lines) is set, that is, when equal attention is paid to tracking the driver's and the automated steering system's target paths, the two controllers' steering angle histories are identical. As a result, the vehicle travels along a path equally displaced from the driver's and the automated system's target paths. When $\rho_{1}=0.8$ and $\rho_{2}=0.2$ are set (dashed lines), that is, when more attention is paid to tracking the driver's straight-line target path, the amplitudes of both controllers' steering angles decrease in comparison to the $\rho_{1}=\rho_{2}=0.5$ case. Reasonably, the vehicle gets closer to the driver's target path. In contrast, when $\rho_{1}=0.1$ and $\rho_{2}=0.9$ (dotted lines), the vehicle gets closer to the automated steering system's lane-change target path.

Fig. 2 (b) displays the influence of lateral displacement error weights $q_{1}^{y}$ and $q_{2}^{y}$. Here $N$ remains 200 steps, i.e. $2.0 \mathrm{~s}, \rho_{1}$ and $\rho_{2}$ are both set to be 0.5 , and the yaw angle error weights $q_{1}^{\psi /}$ and $q_{2}^{\psi}$ and the lateral displacement error integral weights $q_{1}^{y_{\text {int }}}$ and $q_{2}^{y_{\text {int }}}$ are all kept 0 . The $q_{1}^{y}=q_{2}^{y}=0.1$ case which was studied in Fig. 2 is then used as the benchmark (solid lines). It can be seen when the driver's $q_{1}^{y}$ is increased unilaterally to 0.4 (dashed lines), both controllers' steering angles decrease but remain identical. As a result, the vehicle gets closer to the driver's straight-line target path. When the automated steering system's $q_{2}^{y}$ is increased unilaterally to 0.8 (dotted lines), the two controllers' steering angles increase, and the vehicle gets closer to the automated system's lane-change target path.

Fig. 2 (c) exhibits the influence of lateral displacement error integral weights $q_{1}^{y_{\text {int }}}$ and $q_{2}^{y_{\text {int }}}$. In this study, $N$ remains 200 steps, i.e. $2.0 \mathrm{~s}, \rho_{1}$ and $\rho_{2}$ remain 0.5 , and the other path-error weights are set to be $q_{1}^{y}=q_{2}^{y}=0.1$ and $q_{1}^{\psi}=q_{2}^{\psi}=0$. The benchmark case involves $q_{1}^{y_{\text {int }}}=q_{2}^{y_{\text {int }}}=0$ (solid lines), which is identical to the benchmarks used in Fig. 2 (a) and (b). On this basis, when a tiny value of $q_{1}^{y_{\text {itt }}}$ is introduced, that is $q_{1}^{y_{\text {int }}}=1 \mathrm{e}-5$ (dashed lines), both the driver's and automated system's steering angles reduce compared to the benchmark case, and the vehicle converges to the driver's straight-line target path. In order to further examine the effect of penalizing 

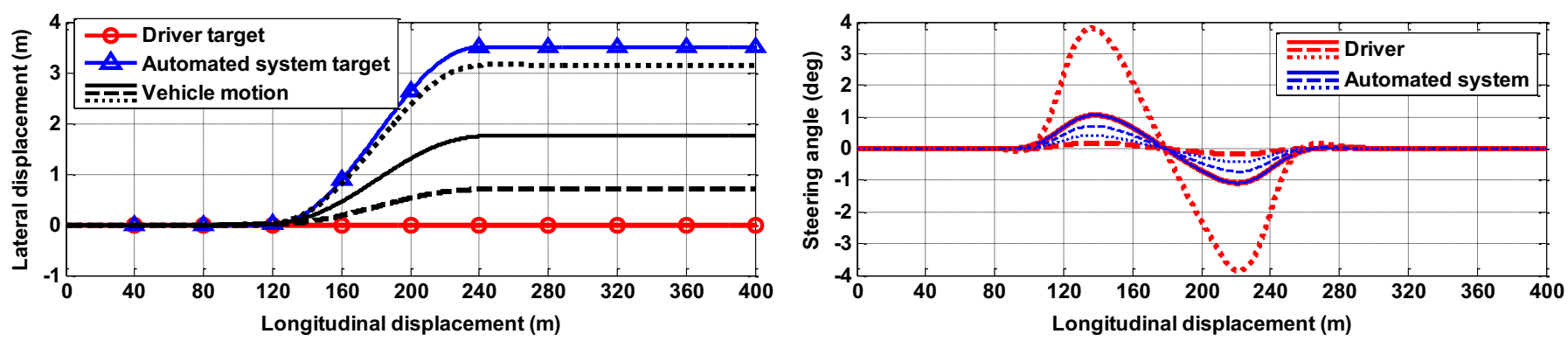

(a)
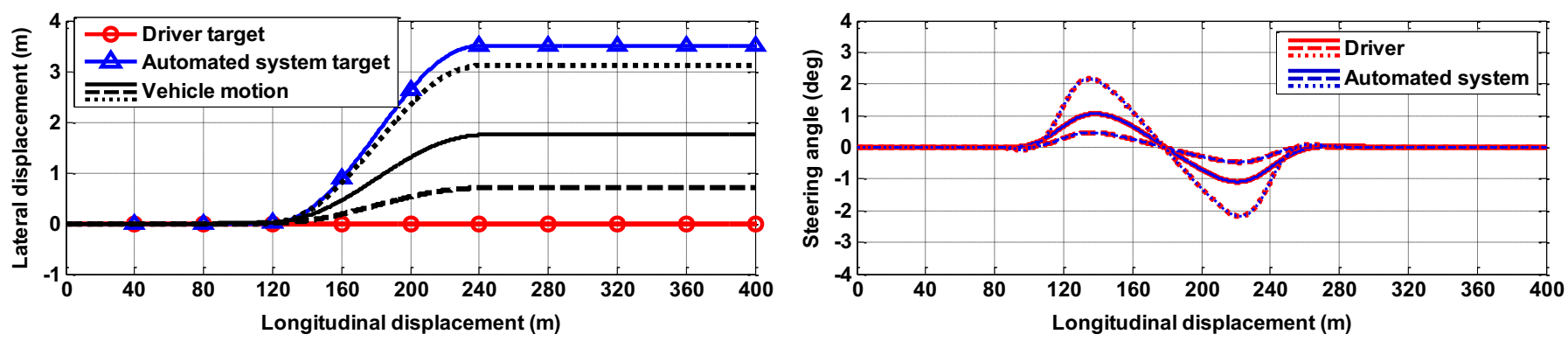

(b)
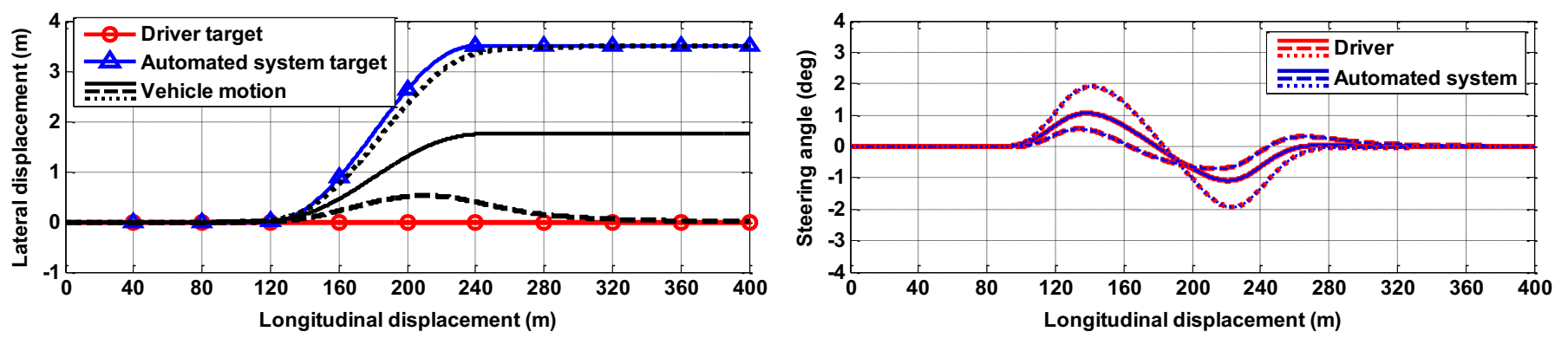

(c)
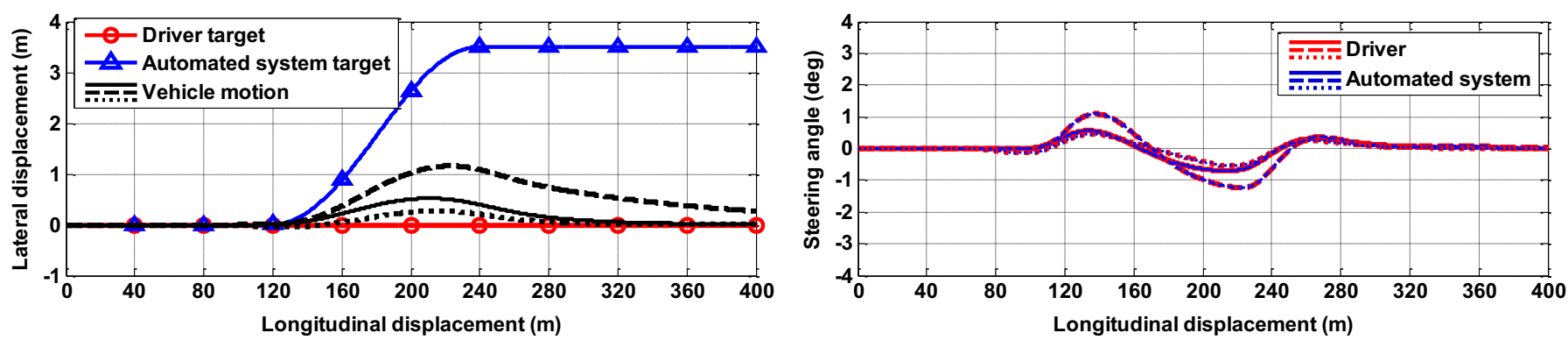

(d)

Fig. 2. Simulated time histories of vehicle lateral displacement (leftward) and driver and automated system steering angles (rightward). Subplot (a) shows the influence of cost function relative weights $\rho_{1}$ and $\rho_{2}$, where solid: $\rho_{1}=\rho_{2}=0.5$, dashed: $\rho_{1}=0.8, \rho_{2}=0.2$, and dotted: $\rho_{1}=0.1, \rho_{2}=0.9$. Subplot (b) shows the influence of lateral displacement error weights $q_{1}^{y}$ and $q_{2}^{y}$, where solid: $q_{1}^{y}=q_{2}^{y}=0.1$, dashed: $q_{1}^{y}=0.4, q_{2}^{y}=0.1$, and dotted: $q_{1}^{y}=0.1, q_{2}^{y}=0.8$. Subplot (c) shows the influence of lateral displacement error integral weights $q_{1}^{y_{\text {int }}}$ and $q_{2}^{y_{\text {int }}}$, where solid: $q_{1}^{y_{\text {int }}}=q_{2}^{y_{\text {int }}}=0$, dashed: $q_{1}^{y_{\text {int }}}=1 \mathrm{e}-5, q_{2}^{y_{\text {int }}}=0$ and dotted: $q_{1}^{y_{\text {int }}}=0, q_{2}^{y_{\text {int }}}=2 \mathrm{e}-5$. Subplot (d) shows the influence of preview horizon $N$, where solid: $N=200$, dashed: $N=100$, and dotted: $N=300$.

lateral displacement error integral, the dashed vehicle lateral displacement shown in Fig. 2 (c) is compared to that shown in Fig. 2 (b). It can be found from Fig. 2 (c) that the introduction of $q_{1}^{y_{\text {iit }}}=1 \mathrm{e}-5$ enables the vehicle to eventually get onto the driver's straight-line target path without experiencing steady-state path-following error. Whereas in Fig. 2 (b) where there is no $q_{1}^{y_{\text {itt }}}$ employed, even increasing $q_{1}^{y}$ from 0.1 to 0.4 , that is, enlarging $q_{1}^{y}$ by four times cannot help with eliminating the vehicle's steady-state path-following error from the driver's straight-line target path. By the same token, the introduction of $q_{2}^{y_{\text {int }}}=2 \mathrm{e}-5$ (dotted lines) causes increase in both the driver's and the automated system's steering angles, and results in the elimination of vehicle path-following error with respect to the automated system's lane-change target path. 
Fig. 2 (d) illustrates the influence of preview horizon $N$. In this study, $\rho_{1}$ and $\rho_{2}$ remains 0.5 , and the path-error weights are set to be $q_{1}^{y}=q_{2}^{y}=0.1, q_{1}^{\psi}=q_{2}^{\psi}=0, q_{1}^{y_{\text {int }}}=1 \mathrm{e}-5$, and $q_{2}^{y_{\text {int }}}=0$. The benchmark case employs $N=200$ steps, i.e. 2.0 $\mathrm{s}$ (solid lines). It can be seen that the vehicle converges to the driver's straight-line target path in this case, mainly due to the introduction of $q_{1}^{y_{\text {itt }}}=1 \mathrm{e}-5$. When the preview horizon $N$ is reduced to 100 steps, i.e. $1.0 \mathrm{~s}$ (dashed lines), the driver's and the automated system's steering angles increase and the vehicle takes longer time to get onto the driver's target path. In contrast, when $N$ is risen to 300 steps, i.e. 3.0 s (dotted lines), the two controller's steering angles decrease and the vehicle converges to the driver's target path more quickly.

\section{CASe Study: COMParison BetWeEn Driver} NON-COOPERATIVE AND COOPERATIVE STEERING STRATEGIES

The numerical analysis presented in the previous section involves a driving scenario where the driver and the automated steering system hold different target paths, and both adopt cooperative Pareto steering strategies. Such a driving scenario is fine for studying the effect of varying control parameters; however, it is less realistic in terms of representing practical driving situations. In fact, a human driver is less likely to adopt a cooperative steering strategy when he/she holds a different goal from the automated steering system in vehicle control. On the other hand, the driver would be more liable to cooperate with the automated system when he/she has developed a feeling that the automated system is attempting to pursue a goal similar to his/her own goal. In light of this, a more realistic driving scenario is designed in this section, and the impact of a driver's adoption of a cooperative Pareto steering strategy, compared to his/her adoption of a non-cooperative Nash steering strategy is studied.

\section{A. Driving Scenario and Target Paths}

The driving scenario employed in this case study is outlined in Table III. It is, in general, a path-following scenario where both the driver and the automated steering system aim at controlling the vehicle to follow their individual target paths. The driving scenario can be divided into two stages according to what particular target path the human driver follows. During the first stage, the driver holds a double-lane-change target path while the automated steering system holds a straight-line target path. Such a conflict in target paths may arise when the driver thinks it would be safe to perform an overtaking maneuver, but the automated system has detected some potential risk from the driver's lane change initiative, e.g., an incoming vehicle in the opposite lane, and thus started its lane keeping control automatically. As a result, the driver would attempt to steer the vehicle to move into the opposite lane while the automated steering system would attempt to pull the vehicle back to its original lane. Consequently, the driver and the automated steering system tend to compete for vehicle directional control. The vehicle, subjected to the steering control of both the driver and the automated steering system, would possibly displace
TABLE III

DRIVING SCENARIO OUTLINE

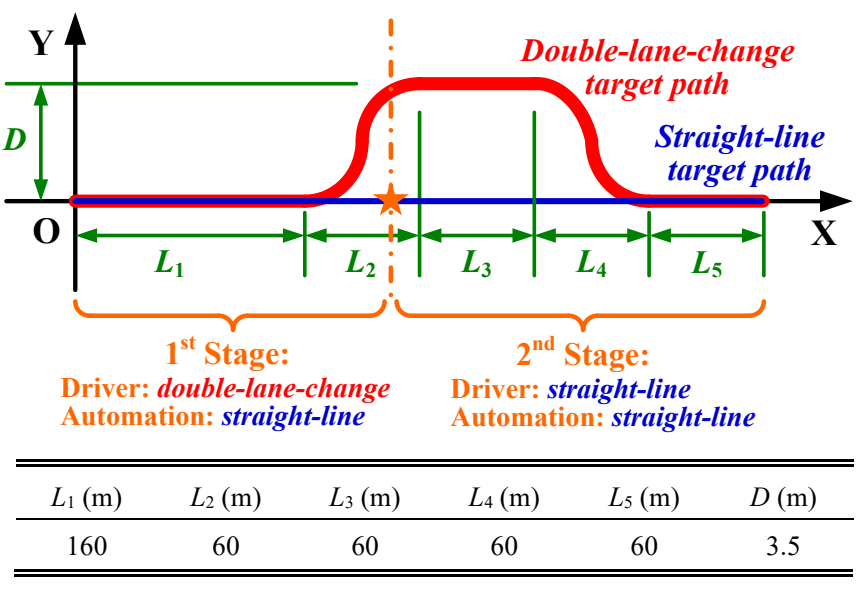

laterally as it travels ahead. Later, the driver may be able to see the incoming vehicle in the opposite lane. Accordingly, the driver may decide to give up his/her initial attempt at lane change but start to apply counter steering so as to get back to the original lane. Such a shift in the driver's goal marks the end of the first stage but the start of the second stage. This instant is schematically marked using a 'star' symbol as shown in Table III. The boundary between the two stages is then marked using a dash-dot line that passes the star symbol. During the second stage, the driver no longer holds the double-lane-change target path but sticks to a straight-line target path that is identical to the target path of the automated steering system.

\section{B. Steering Strategies}

The automated steering system concerned in this study uses a steering strategy of the non-cooperative Nash form [18]. Such a steering strategy partially neutralizes the effect of the driver's steering control so that possible adverse effects due to human errors can be mitigated. Automated steering control that follows similar design concept has been studied and validated in several publications, e.g. [30] and [31].

The driver is assumed to use a non-cooperative Nash steering strategy to interact with the automated steering system during the first stage. Derivation of the driver's non-cooperative Nash steering strategy was formulated in detail in [18]. Here a recap of the key points of the derivation is provided below.

\section{1) Driver's cost function in a non-cooperative game}

In a non-cooperative game, the driver focuses on pursuing his/her individual interest, that is to minimize his/her individual path-following error $\mathbf{E}_{1}(k)$. Hence, the driver's cost function can be expressed as:

$$
J_{1}(k)=\mathbf{E}_{1}(k)^{\mathrm{T}} \mathbf{Q}_{1} \mathbf{E}_{1}(k)+\mathbf{U}_{1}(k)^{\mathrm{T}} \mathbf{U}_{1}(k)
$$

By comparing the driver's non-cooperative cost function (25) to the driver's cooperative cost function (4), it can be found that in a non-cooperative game, the driver does not take into account the automated system's path-following interest $\mathbf{E}_{2}(k)$. This is the key difference between a non-cooperative game and a 
cooperative game. In other words, this reflects the fundamental difference between a cooperative driver and a noncooperative driver. It should be noted that a cooperative driver does not necessarily require their own path-following interest $\mathbf{E}_{1}(k)$ to be in consistent with the automated steering system's path-following interest $\mathbf{E}_{2}(k)$. In theory in a cooperative case $\mathbf{E}_{1}(k)$ and $\mathbf{E}_{2}(k)$ can be different or even distinct from each other, e.g. the driver attempts to follow a straight-line path while the automated steering system attempts to follow a lane-change target path, as discussed in the previous section. Such a concept of cooperation is consistent with corresponding definitions proposed in many other publications, e.g. [32].

\section{2) Driver's prediction equation in a non-cooperative game}

In a non-cooperative game, the driver does not use the global prediction equation (10). Instead, the driver uses his/her individual prediction equation (8).

\section{3) Driver's non-cooperative Nash steering strategy}

By optimizing the driver's non-cooperative cost function (25) subject to the driver's individual prediction equation (8), the driver's non-cooperative Nash steering strategy can be finally obtained, as shown in (26):

$$
\delta_{1}^{\prime}(k)=\mathbf{K}_{1}^{\prime}(1,:)\left\{\begin{array}{c}
\mathbf{x}(k) \\
\mathbf{R}_{1}(k) \\
\mathbf{R}_{2}(k)
\end{array}\right\}
$$

By comparing the driver's non-cooperative Nash steering strategy (26) to his/her cooperative Pareto steering strategy (23), it can be seen that they are similar in format. However, the $\mathbf{K}_{1}{ }^{\prime}$ appearing in the non-cooperative Nash steering strategy (26) is irrelevant to the cost function relative weights $\rho_{1}$ or $\rho_{2}$.

During the first stage as depicted in Table III, the assumption that the driver uses a non-cooperative Nash steering strategy to interact with the automated system is thought reasonable. This is mainly due to the situation that the driver choses to follow a target path which is completely different from the target path of the automated system. Accordingly, the driver may naturally move to the Nash equilibrium at which the driver's interest in terms of tracking the double-lane-change target path can be maximized. On the other hand, it would be difficult to imagine that the driver could develop a cooperative Pareto strategy over this stage, which requires the driver to consider the interest of the automated system in terms of its path-following control.

Later when the driver enters the second stage, that is, when the driver decides to give up his/her lane change aspiration but start to follow a straight-line target path, the probability that the driver develops a cooperative Pareto steering strategy may rise. This is primarily because during the second stage the driver intends to track a straight-line target path which is identical to that of the automated system. The alignment of the driver's target path with that of the automated system may affect the driver to account for the automated system's path-following control. Alternatively, the driver may continue using the non-cooperative Nash strategy that he/she has been using through the first stage.
In a nutshell, the driver's possible steering behavior during the two stages may be summarized as:

1) During the first stage, the driver has a double-lane-change target path. During this stage, the assumption that the driver uses a non-cooperative Nash steering strategy is reasonable; whereas the assumption that the driver uses a cooperative Pareto steering strategy is less reasonable.

2) During the second stage, the driver has a straight-line target path. During this stage, either the assumption that the driver continues to adopt a non-cooperative Nash steering strategy or that the driver develops a cooperative Pareto steering strategy could be rational.

It is noteworthy here that during the second stage the proposition that the driver holds a straight-line target path identical to the automated steering system's target path does not conflict with the authors' assumption that the driver adopts a non-cooperative Nash strategy. In other words, the driver in theory can still use a non-cooperative steering strategy to interact with the automated system even when the two controllers have identical target path. This is supported by the fact that a non-cooperative game does not necessarily require the game players to have conflicting interest but mainly requires them to focus pursuing their interest individually [10]. By transferring such requirements into the case discussed above, the driver's adoption of a non-cooperative steering strategy suggests that the driver focuses on pursuing the driver's individual interest in term of path tracking without considering the automated steering system's interest. The driver's adoption of a non-cooperative steering strategy is irrelevant to whether the driver's and the automated steering system's path-tracking interest are identical or distinct.

\section{Simulation Results}

Based on the formulation made above, a comparative study is carried out aiming at inspecting the impact of different driver steering strategies on the driver's and the automated system's steering angles and vehicle directional responses. The switch from the first stage to the second stage is set to be triggered when the vehicle reaches a lateral displacement of $1.0 \mathrm{~m}$, resulting from the steering interaction between the driver and the automated system during the first stage. Such a lateral-displacement-based trigger is practical since the driver's view of the opposite lane will become clearer as the vehicle moves more in the lateral direction. Accordingly, the driver at some point may be able to see the incoming vehicle in the opposite lane. When this happens, the driver will give up his/her attempt at lane change but steer the vehicle back to the original lane. The simulation time step, vehicle longitudinal speed and vehicle parameters are kept identical to those employed in Section III. The preview horizon $N$ is set to be 100 steps, i.e. $1.0 \mathrm{~s}$. On this basis, simulation results obtained from the driver adopting a cooperative Pareto steering strategy during the second stage are compared to those obtained from the driver using a non-cooperative Nash steering strategy during the second stage. Specifically, the comparison is made under three sets of control parameters: 

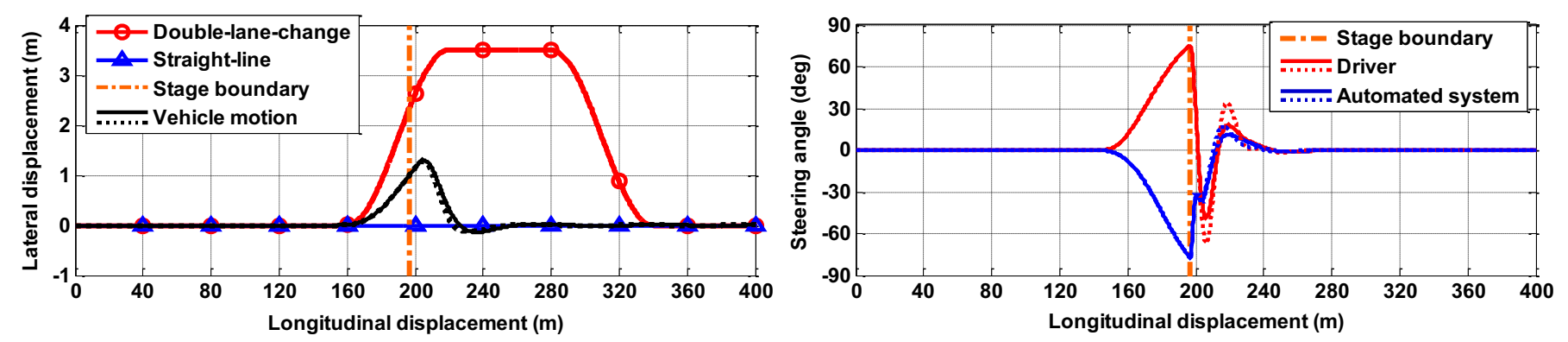

(a)
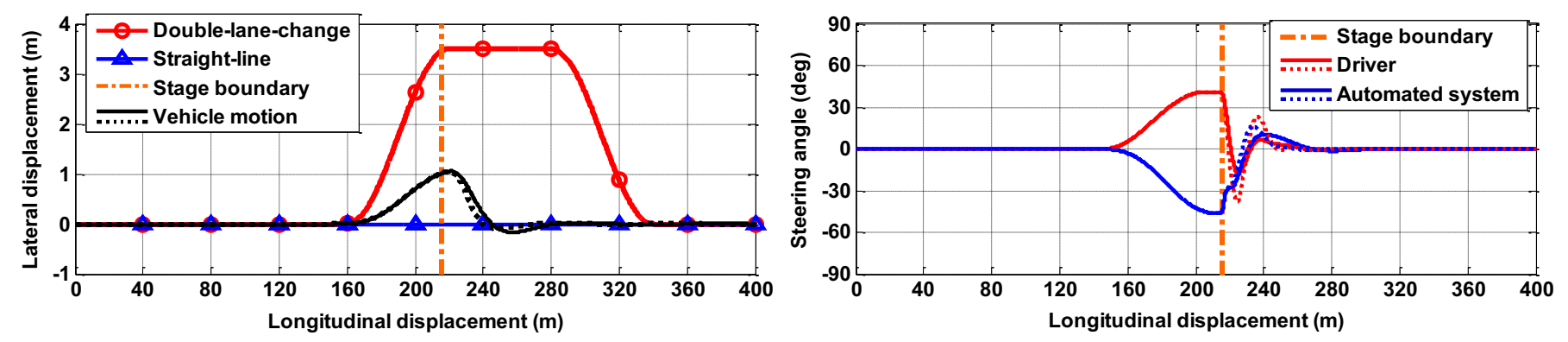

(b)
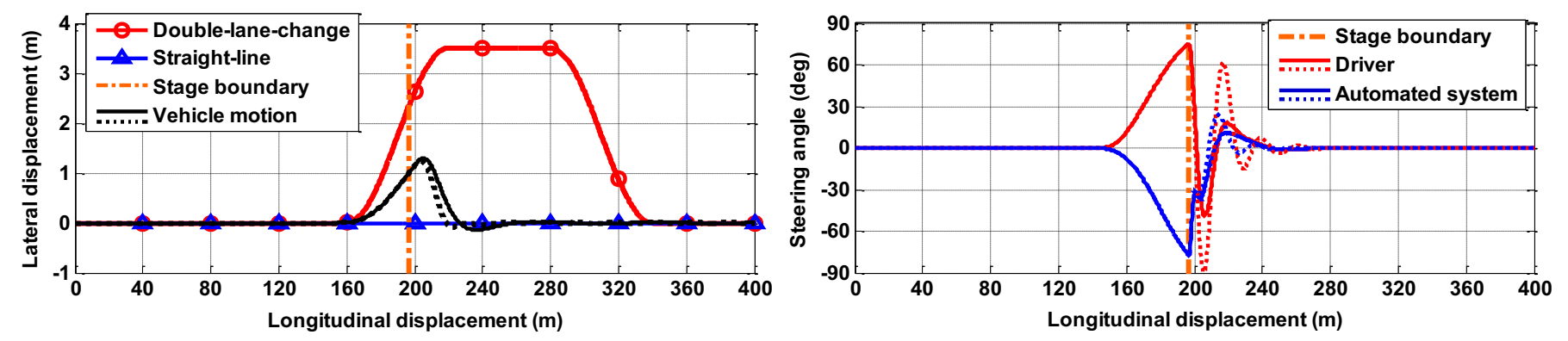

(c)

Fig. 3. Simulated time histories of vehicle lateral displacement (leftward) and driver and automated system steering angles (rightward). Subplot (a) shows the results from parameter Set A, where solid: driver using non-cooperative Nash strategy during the second stage, dotted: driver using cooperative Pareto strategy during the second stage. Subplot (b) shows the results from parameter Set B, where solid: driver using non-cooperative Nash strategy during the second stage, dotted: driver using cooperative Pareto strategy during the second stage. Subplot (c) shows the results from parameter Set C, where solid: driver using non-cooperative Nash strategy during the second stage, dotted: driver using cooperative Pareto strategy during the second stage.

Set A: $\rho_{1}=\rho_{2}=0.5, q_{1}^{y}=0.6$, and $q_{2}^{y}=0.4$,

Set B: $\rho_{1}=\rho_{2}=0.5, q_{1}^{y}=0.2$, and $q_{2}^{y}=0.4$,

Set C: $\rho_{1}=0.3, \rho_{3}=0.7, q_{1}^{y}=0.6$, and $q_{2}^{y}=0.4$.

All the rest path-error weights of the driver and of the automated steering system are kept 0 .

Fig. 3 (a) illustrates the simulation results from parameter Set A. The boundary between the two stages is marked using a dash-dot line vertical to the horizontal axis in this figure. During the first stage, that is, at the left-hand-side of the dash-dot boundary line, both the driver and the automated steering system adopt non-cooperative Nash steering strategies. The driver is set to pursue the double-lane-change target path while the automated system is set to follow the straight-line target path. It can be seen that the driver and the automated system steer in opposite directions, and the vehicle starts to deviate from its original lane. When the vehicle reaches a lateral displacement of $1.0 \mathrm{~m}$, the driver is assumed to realize the risk of his/her lane change maneuver and thus changes his/her target path from double-lane-change to straight-line. This change in the driver's target path marks the start of the second stage. As it was explained previously, during the second stage, the driver may adopt either a non-cooperative Nash or a cooperative Pareto steering strategy to interact with the automated steering system. Results from the driver adopting the non-cooperative strategy during this stage are presented in solid lines while those from the driver adopting the cooperative Pareto strategy are shown in dotted lines for comparison. It can be seen that compared to the case where the driver adopts a non-cooperative Nash strategy, the case where the driver uses a cooperative Pareto strategy involves larger driver steering angles towards moving back to the vehicle's original lane. Specifically, the driver's steering angle is $49.3 \mathrm{deg}$ when it reached the first peak under the non-cooperative Nash strategy. This steering angle is shown in Fig. 3 (a) as a negative value, 
which indicates the driver steering towards direction of the original lane. In contrast, the driver's first peak steering angle under the cooperative Pareto strategy is $66.7 \mathrm{deg}$ (shown in Fig. 3 (a) as a negative value). Accordingly, the vehicle controlled under the driver's adoption of a cooperative Pareto steering strategy first reached the straight-line target path at a longitudinal displacement of $222.4 \mathrm{~m}$, compared to that of $226.4 \mathrm{~m}$ under the driver's adoption of non-cooperative Nash steering strategy. Such a 4-meter difference in longitudinal direction suggests that the driver's adoption of a cooperative Pareto strategy enables the vehicle to return to the straight-line target path $0.2 \mathrm{~s}$ swifter than the driver's adoption of a noncooperative Nash strategy. Soon after, the driver's and the automated steering system's steering angles converge to zero, and the vehicle travels straight ahead in its original lane. The differences between the steering angle time histories of the driver's cooperative Pareto and his/her non-cooperative Nash stem from the differences in the prediction equations and cost functions used for deriving the two steering strategies.

Fig. 3 (b) exhibits the simulation results from parameter Set $\mathrm{B}$, where the driver's lateral displacement error weight $q_{1}^{y}$ is significantly reduced compared to that in Set A. It can be seen from this figure that the characteristics of the interaction between the driver and automated steering system are generally similar to those shown in Fig. 3 (a): the driver and the automated system steers counter to each other during the first stage while their steering angles converge to zero in the second stage. However, there exist one key difference between the results shown in Fig. 3 (b) and Fig. 3 (a): since the driver's weight $q_{1}^{y}$ is reduced, the driver would have less capacity to steer the vehicle to follow his/her double-lane-change target path during the first stage. Due to this fact, the amplitudes of the driver's and the automated system's steering angles exhibited in the first stage, i.e. at the left side of the dash-dot boundary are obviously lower than those shown in Fig. 3 (a). Specifically, under parameter Set B, the driver's peak steering angle during the first stage is 41.0 deg while under parameter Set $\mathrm{A}$, the corresponding value reaches $74.9 \mathrm{deg}$. Accordingly, in fig. 3(b) the vehicle reaches the $1.0 \mathrm{~m}$ lateral displacement later than in Fig. 3 (a). Besides this key difference, it is also noteworthy that in both Fig. 3 (a) and (b), that is, under both parameter Sets A and $\mathrm{B}$, the driver's adoption of a cooperative Pareto steering strategy in the second stage (as shown in dashed lines) brings about larger driver steering angles compared to the driver's adoption of a non-cooperative Nash strategy. This enables the vehicle to move back to straight-line driving more quickly.

Fig. 3 (c) displays the simulation results from parameter Set $\mathrm{C}$, where unbalanced cost function weights $\rho_{1}=0.3$ and $\rho_{2}=0.7$ are employed. Such setting suggests that when the driver uses the cooperative Pareto steering strategy, the driver accounts more for the automated steering system's control behavior. In other words, the driver with such cost function weights tends to be more cooperative from the automated steering system's point of view. It can be seen that compared to parameter Set A (Fig. 3 (a)) where balanced cost function weights are used, the driver in this unbalanced situation tends to steer more intensively when he/she adopts a cooperative Pareto strategy during the second stage (see dashed lines in the rightward subplot in Fig. 3 (c)). Specifically, the driver's steering angle reaches $89.4 \mathrm{deg}$ at its first peak (shown in Fig. 3 (c) as a negative value), compared to the corresponding 66.7 deg obtained under parameter Set A (Fig. 3 (a)). This in turn enables the vehicle to return to its original lane at a longitudinal displacement of $218.6 \mathrm{~m}$, which is more quickly compared to the $222.4 \mathrm{~m}$ observed under parameter Set A.

\section{CONCLUSION}

This paper concerned modelling a human driver's interaction with an automated driving system using cooperative game theory. The interaction problem was discussed in the context of a driver controlling a vehicle equipped with an automated steering system, where the driver and the automated system had individual target paths, and were able to apply steering angles to the vehicle independently. The distributed Model Predictive Control approach was adopted to analytically derive the driver's and the automated steering system's steering strategies in a Pareto equilibrium sense, namely their cooperative Pareto steering strategies.

Numerical analysis was performed to examine the influence of key parameters in Pareto steering strategies on the driver's and the automated steering system's steering angles and vehicle lateral response. It was found that when the driver is interacting with an automated steering system that held a different target path, the driver could improve his/her path-following capability by means of i) increasing his/her penalization on vehicle lateral deviation, ii) enlarging his/her preview horizon, and iii) putting more emphasis on pursuing his/her own interest in the driver's cooperative cost function.

A comparative case study was then carried out to inspect the difference between the driver's adoption of a non-cooperative Nash steering strategy and the driver's use of a cooperative Pareto steering strategy in the same driving scenario. It was found that the driver's adoption of a cooperative Pareto steering strategy lead to different steering angle histories and vehicle lateral motions from those resulted from the driver's adoption of a non-cooperative Nash steering strategy. Specifically, the driver's adoption of Pareto strategy resulted in a reinforcement of the driver's steering angle control, which in turn enabled the vehicle to return from a risky lane-change maneuver back to a safer straight-line travelling quickly.

In future work, experiments using a driving simulator will be conducted to measure human driver steering behavior from a large number of test drivers with different driving experience. The cooperative Pareto steering strategy proposed in this paper will be fit to all measured driver steering behavior for validation. Key driver control parameters involved in the cooperative Pareto steering strategy will be identified through a proper system identification approach. The identified driver control parameters can then be built into automated steering technology to enable the technology to take into account human 
drivers' real-life steering control behavior and to achieve more effective shared control between driver and automation.

\section{REFERENCES}

[1] T. J. Gordon and M. Lidberg, "Automated driving and autonomous functions on road vehicles," Veh. Syst. Dyn., vol. 53, no. 7, pp. 958-994, Jul. 2015.

[2] D. J. Fagnant and K. Kockelman, "Preparing a nation for autonomous vehicles: Opportunities, barriers and policy recommendations," Transp. Res. Part A: Policy Pract., vol. 77, pp. 167-181, Jul. 2015.

[3] A. Nunes, B. Reimer and J. F. Coughlin, "People must retain control of autonomous vehicles", Nature, vol. 556, pp. 169-171, Apr. 2018.

[4] W. Klier, G. Relmann, and W. Reinelt, "Concept and functionality of the Active Front Steering system," Soc. Automotive Eng., Warrendale, PA, USA, SAE Technical Paper 2004-21-0073, Oct. 2004.

[5] H. Guo, L. Song, J. Liu, F. Wang, D. Cao, H. Chen, C. Lv, and P. C. Luk, "Hazard-evaluation-oriented moving horizon parallel steering control for driver-automation collaboration during automated driving," IEEE/CAA J. Autom. Sinica, vol. 5, no. 6, pp. 1062-1073, Nov. 2018.

[6] D. A. Abbink, T. Carlson, M. Mulder, J. C. F. de Winter, F. Aminravan, T. L. Gibo, and E. R. Boer, "A topology of shared control systems finding common ground in diversity," IEEE Trans. Human-Mach. Syst., vol. 48, no. 5, pp. 509-525, Oct. 2018.

[7] G. Guo and Q. Wang, "Fuel-efficient en route speed planning and tracking control of truck platoons," IEEE Trans. Intell. Transp. Syst., to be published.

[8] L. Chen, X. Hu, W. Tian, H. Wang, D. Cao, and F. Wang, "Parallel planning: a new planning framework for autonomous driving," IEEE/CAA J. Autom. Sinica., vol. 6, no. 1, pp. 236-246, Jan. 2019.

[9] T. Başar and G. J. Olsder. Dynamic Noncooperative Game Theory, 2nd ed. New York, NY, USA: Academic, 1995, ch. 1.

[10] Y. Shoham and K. Leyton-Brown, Multiagent Systems: Algorithmic, Game-theoretic, and Logical Foundations. Cambridge, U.K.: Cambridge Univ. Press, 2009, ch. 3.

[11] W. Ma and H. Peng, "Worst-case vehicle evaluation methodology examples on truck rollover/jackknifing and active yaw control systems," Veh. Syst. Dyn., vol. 32, no. 4-5, pp. 389-408, May 1999.

[12] S. H. Tamaddoni, S. Taheri, and M. Ahmadian, "Optimal preview game theory approach to vehicle stability controller design," Veh. Syst. Dyn., vol. 49, no. 12, pp. 1967-1979, Dec. 2011.

[13] C. Dextreit and I. V. Kolmanovsky, "Game theory controller for hybrid electric vehicles," IEEE Trans. Control Syst. Technol., vol. 22, no. 2, pp. 652-663, Mar. 2014.

[14] M. Flad, L. Fröhlich, and S. Hohmann, "Cooperative shared control driver assistance systems based on motion primitives and differential games," IEEE Trans. Human-Mach. Syst., vol. 47, no. 5, pp. 711-722, Oct. 2017.

[15] X. Na and D.J. Cole, "Application of open-loop Stackelberg equilibrium to modelling a driver's interaction with vehicle active steering control in obstacle avoidance," IEEE Trans. Human-Mach. Syst., vol. 47, no. 5, pp. 673-685, Oct. 2017.

[16] X. Ji, Y. Liu, X. Na, and Y. Liu, "Research on interactive steering control strategy between driver and AFS in different game equilibrium strategies and information patterns," Veh. Syst. Dyn., vol. 56, no. 9, pp. 1344-1374, Feb. 2018.

[17] X. Ji, K. Yang, X. Na, C. Lv, and Y. Liu, "Shared steering torque for lane change assistance: a stochastic game-theoretic approach," IEEE Trans. Ind. Electron., vol. 66, no. 4, pp. 3093-3105, Apr. 2019.

[18] X. Na and D. J. Cole, "Game-theoretic modeling of the steering interaction between a human driver and a vehicle collision avoidance controller," IEEE Trans. Human-Mach. Syst., vol. 45, no. 1, pp. 25-38, Feb. 2015.

[19] C. W. Rosier and D. J. Cole, "Model predictive control to represent interaction of driver and active accelerator pedal," presented at the Int. Symp. Advanced Vehicle Control, Munich, Germany, 2016.

[20] H. Guo, D. Cao, H. Chen, C. Lv. H. Wang, and S. Yang, "Vehicle dynamic state estimation: state of the art schemes and perspectives," IEEE/CAA J. of Autom. Sinica, vol. 5, no. 2, pp. 418-431, Mar. 2018.

[21] Y., Xing, C. Lv, H. Wang, D. Cao, E. Velenis, and F. Wang, "Driver activity recognition for intelligent vehicles," IEEE Trans. Veh. Tech., in press, 2019.

[22] J. F. Nash. "Non-cooperative games", Ann. Math., vol. 54, pp. 286-295, 1951.
[23] D. J. Cole, "Occupant-vehicle dynamics and the role of the internal model," Veh. Syst. Dyn., vol. 56, no. 5, pp. 661-688, Dec. 2018.

[24] A. Y. Ungoren and H. Peng, "An adaptive lateral preview driver model," Veh. Syst. Dyn., vol. 43, no. 4, pp. 245-259, Apr. 2005.

[25] S. D. Keen and D. J. Cole, "Bias-free identification of a linear modelpredictive steering controller from measured drier steering behaviour," IEEE Trans. Syst., Man, Cybern. B, Cybern., vol. 42, no. 2, pp. 434-443, Apr. 2012.

[26] J. B. Rawlings and D. Q. Mayne, Model Predictive Control: Theory and Design. New York: NY, USA: Nob Hill, 2009, ch. 6.

[27] X. Na, "Game theoretic modelling of a driver's steering interaction with active steering", Ph.D. dissertation, Dept. Eng., Univ. Cambridge, Cambridge, U.K., 2014.

[28] J. M. Maciejowski, Predictive Control: With Constraints. London, U.K.: Prentice-Hall, 2002, ch. 1.

[29] A. Odhams, "Identification of driver steering and speed control", Ph.D. dissertation, Dept. Eng., Univ. Cambridge, Cambridge, U.K., 2006.

[30] S. J. Anderson, J. M. Walker, and K. Iagnemma, "Experimental performance analysis of a homotopy-based shared autonomy framework," IEEE Trans. Human-Mach. Syst., vol. 44, no. 2, pp. 190-199, Apr. 2014.

[31] S. M. Erlien, S. Fujita, and J. C. Gerdes, "Shared steering control using safe envelopes for obstacle avoidance and vehicle stability," IEEE Trans. Intell. Transp. Syst., vol. 17, no. 2, pp. 441-451, Feb. 2016.

[32] J. M. Hoc, "Towards a cognitive approach to human-machine cooperation in dynamic situations," Int. J. Human-Computer Studies, vol. 54, no. 4, pp. 509-540, 2001.

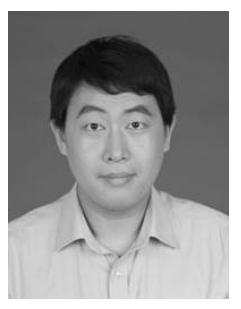

Xiaoxiang Na received the B.Sc. and M.Sc. degrees in automotive engineering from the College of Automotive Engineering, Jilin University, China, in 2007 and 2009, respectively. He received the Ph.D. degree in driver -vehicle dynamics from the Department of Engineering, University of Cambridge (CUED), U.K. in 2014, where he is currently a Senior Research Associate.

His main research interests include driver-vehicle dynamics and vehicle in-service monitoring.

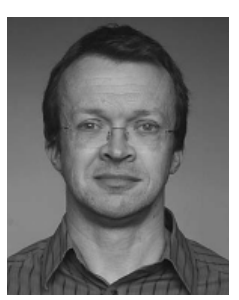

David J. Cole received the B.A. degree in engineering and the Ph.D. degree in vehicle dynamics from the Department of Engineering, University of Cambridge (CUED), U.K. in 1985 and 1990, respectively.

From 1990 to 1996, he undertook his postdoctoral research in heavy vehicle dynamics at CUED, where he is currently a University Reader. From 1996 to 2000, he was a Lecturer with the University of Nottingham, U.K. In 2000, he returned to CUED to take up his present position. His main research interests include driver-vehicle dynamics. 